(6)

\section{OPEN ACCESS}

${ }^{1}$ Clinical Effectiveness Unit, Royal College of Surgeons of England, London, UK

${ }^{2}$ South West Cleft Unit, Frenchay Hospital, North Bristol NHS Trust, Bristol, UK ${ }^{3}$ Department of Health Services Research and Policy, London School of Hygiene and Tropical Medicine, London, UK

\section{Correspondence to}

Dr Kate J Fitzsimons, Clinical Effectiveness Unit, Royal College of Surgeons of England, 35-43 Lincoln's Inn Fields, London WC2A 3PE, UK kfitzsimons@rcseng.ac.uk

Received 23 April 2013 Revised 23 July 2013 Accepted 26 July 2013 Published Online First 22 August 2013

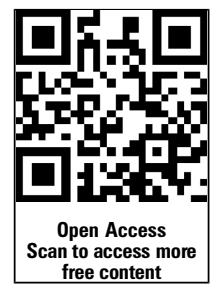

To cite: Fitzsimons KJ, Copley LP, Deacon SA, et al. Arch Dis Child

2013;98:970-974.

\title{
Hospital care of children with a cleft in England
}

\author{
Kate J Fitzsimons, ${ }^{1}$ Lynn P Copley, ${ }^{1}$ Scott A Deacon, ${ }^{1,2}$ Jan H van der Meulen ${ }^{1,3}$
}

\section{ABSTRACT \\ Objective To analyse hospital admissions in the first}

2 years of life among children with cleft lip and/or palate in England.

Design Analysis of national administrative data of hospital admissions.

Setting National Health Service hospitals.

Patients Patients born alive between 1997 and 2008 who underwent surgical cleft repair.

Outcome measures Number of admissions, including the birth episode, and days spent in hospital were examined. Children were analysed according to cleft type and whether or not they had additional congenital anomalies.

Results 10892 children were included. In their first 2 years, children without additional anomalies $(n=8482)$ had on average 3.2 admissions and 13.2 days in hospital, which varied from 2.6 admissions and 9.2 days with cleft lip to 4.7 admissions and 19.7 days with bilateral cleft lip and palate (BCLP). Children with additional anomalies $(n=2410)$ had on average 6.7 admissions and 51.4 days in hospital, which varied from 6.4 admissions and 48.5 days with cleft palate to 8.8 admissions and 67.5 days with BCLP. The mean number and duration of cleft-related admissions was similar in children without ( 1.6 admissions and 6.4 days) and in those with additional anomalies (1.5 admissions and 8.5 days). $35.2 \%$ of children without additional anomalies had at least one emergency admission, whereas the corresponding figure was $67.3 \%$ with additional anomalies.

Conclusions The burden of hospital care in the first 2 years of life varied according to cleft type and presence of additional anomalies. However, cleft-specific hospital care did not differ between children with and without additional anomalies.

\section{INTRODUCTION}

Craniofacial abnormalities are among the most common of all birth defects. ${ }^{1}$ In the UK, about 1 in 700 live-born children has a cleft lip and/or palate. $^{2}{ }^{3}$ Successful management of patients born with a cleft requires multidisciplinary and highly specialised surgical and non-surgical treatment from birth until adulthood. ${ }^{4}$

Recently published national data revealed that $75 \%$ of primary lip repairs are performed between 3 months and 6 months of age, while $86 \%$ of primary palate repairs are performed between 6 months and 2 years of age. ${ }^{5}$ However, the burden of hospital care among these children, specifically in terms of the number of admissions and total time spent in hospital, is currently unknown. Factors such as the type of cleft and whether a child has additional anomalies are likely to influence the hospital admissions and the length of stay.

\section{What is already known on this topic}

- Children with a cleft require surgery to repair the abnormality within the first 2 years of life.

- Since 2006, cleft services in England have been centralised to 13 hospital cleft treatment sites, which provide primary repair surgery for all children with a cleft.

\section{What this study adds}

- This study has quantified hospital cleft care, in terms of admissions and total length of stay, in England during the first 2 years of life.

- Large differences in total time in hospital were revealed between children without and children with additional anomalies and between the four main cleft types.

- Total time spent in hospital for cleft-related procedures before the age of 2 years reduced between 1997 and 2008 among children without additional anomalies.

Quantifying the burden of hospital care is useful for those commissioning and planning cleft services and for counselling parents and carers. A survey demonstrated that what parents want most of all is information about surgery during the first counselling session after diagnosis. ${ }^{6}$

In this article, we describe hospital admissions in the first 2 years of life because the majority of primary cleft repair surgery occurs before the age of 2 years. ${ }^{25}$ Hospital care, in terms of number of admissions and total length of stay, is presented according to cleft type and whether or not the children have additional congenital anomalies.

\section{METHODS}

Data source

Data were extracted from the Hospital Episode Statistics (HES) database, which contains records of all admissions to National Health Service (NHS) hospitals in England. ${ }^{7}$ Diagnostic information is coded using the International Classification of Diseases 10 th revision (ICD-10), and procedure information is classified according to codes from the Classification of Surgical Operations and Procedures 4th Revision (OPCS-4).

\section{Patients}

Patients born between 1 January 1997 and 31 December 2008 were included if they had at least 
one HES record with a diagnosis code for cleft lip and/or palate (ICD-10 codes Q35, Q36, Q37) as well as a record with a procedure code for a primary cleft repair (OPCS-4 codes F031, F291). Hospital episodes up to 31 January 2011 were assessed. Patients who were identified in HES as 'private' and whose postcode was 'unavailable/not applicable' were excluded from analyses as these patients were likely to live outside England, and as a result, their care does not reflect typical cleft care for patients who reside in England.

\section{Hospital admissions and time in hospital}

The total number of hospital admissions (including the birth episode) and the total number of days in hospital before the age of 2 years were identified and included, regardless of the age at discharge.

Admissions for cleft-related procedures were assessed to determine what proportion of all admissions these represented. An admission was defined as 'cleft-related' if at least one of 24 specific procedure codes relating to the ear, respiratory tract or mouth (see online supplementary appendix1) was performed during a particular admission.

The proportion of admissions that were reported in HES as 'emergency' was determined by the reported method of admission, of which there are five options: elective, emergency, birth, non-emergency transfer and unknown. To determine the proportion of children with an emergency readmission that was likely to be cleft-related, we examined emergency admissions within 14 days of discharge after a primary cleft repair procedure, as defined by OPCS-4 codes F031 and F291.

\section{Additional anomalies}

Children were defined as having additional anomalies if any of their episode records had at least one of 33 ICD-10 diagnostic codes (see online supplementary appendix 2) representing congenital malformations and chromosomal abnormalities, in any one of the diagnosis code fields of a HES record.

\section{Cleft type}

Clefts were grouped as cleft lip only (CL), cleft palate only (CP), unilateral cleft lip and palate (UCLP) or bilateral cleft lip and palate (BCLP) according to the presence of selected procedure codes (OPCS-4) and/or diagnosis codes (ICD-10) in any of the available HES records. A stepwise, hierarchical approach was employed. First, the cleft repair procedure codes (F03, F29, F32) were used to identify three cleft type groups: CL, CP, cleft lip and palate (CLP). Second, the diagnosis code was used to distinguish between UCLP and BCLP cases in the CLP group.

\section{Socio-demographic factors}

The Index of Multiple Deprivation, which is based on postcode of residence, was used to assess the patients' socio-economic background. ${ }^{8}$ Ethnicity was assessed according to the HES field 'ethnos', which comprises 18 ethnic categories, which for the purpose of our analyses were grouped into six group categories (white, mixed, Asian, black, other, and unknown).

\section{Analyses}

A descriptive statistical approach was followed, and means, SDs and medians were calculated according to cleft type and the presence or absence of additional congenital anomalies. Linear regression was used to assess temporal trends in total length of stay. All analyses were performed in STATA V.11.

\section{RESULTS}

A total of 10892 children, born between 1 January 1997 and 31 December 2008 with a cleft, were identified. Of these children, $2410(22.1 \%)$ had additional anomalies (table 1). The proportion of children with additional anomalies varied from $7.4 \%$ (187/2529) among children with CL to 35.1\% (1827/ 5205) among those with CP. The children had a total of 43335 admissions, including the birth admission, and spent a total of 235442 days in hospital before they were 2 years old. Neither socio-economic deprivation nor ethnicity was associated with the number of admissions or total time spent in hospital before 2 years of age.

\section{Admissions and time in hospital}

The mean number of all-cause admissions and total time spent in hospital did not change significantly for births between 1997 and 2008. Children without additional anomalies had on average 3.2 admissions and spent 13.2 days in hospital before their second birthday (table 1). Excluding the birth episode, children spent 9.2 days in hospital. Corresponding figures for children with additional anomalies are 6.7 admissions and 51.4 days in hospital (table 1). Excluding the birth episode, they spent on average 38.8 days in hospital. Admissions and days in hospital appeared to be correlated with the 'severity' of the cleft type, both in children without and in those with additional anomalies (table 1). The mean time spent in hospital is always larger than the median value, especially in children with additional anomalies, which corresponds to some children staying in hospital for extended periods of time (please note the large SDs).

\section{Cleft-related procedure admissions}

Unlike all-cause admission time, a significant reduction in overall length of stay for cleft-related procedures was observed over the 12-year period among children without additional anomalies $(\mathrm{p}<0.001)$. Total time in hospital decreased from 6.8 days for children born in 1997 to 5.3 days for those born in 2008. There was no significant temporal trend among children

Table 1 Total number of all-cause admissions and days in hospital for (A) non-syndromic and (B) syndromic cleft patients in the first 2 years of life, according to cleft type; year of birth 19972008

\begin{tabular}{|c|c|c|c|c|c|c|c|}
\hline \multirow[b]{2}{*}{ Cleft type } & \multirow[b]{2}{*}{$\mathrm{N}^{*}$} & \multicolumn{3}{|c|}{$\begin{array}{l}\text { Total number of } \\
\text { admissions }\end{array}$} & \multicolumn{3}{|c|}{$\begin{array}{l}\text { Total number of days in } \\
\text { hospital }\end{array}$} \\
\hline & & Mean & (SD) & Median & Mean & (SD) & Median \\
\hline \multicolumn{8}{|c|}{ (A) Non-syndromic patients } \\
\hline $\mathrm{CL}$ & 2342 & 2.6 & (1.7) & 2 & 9.2 & $(14.5)$ & 7 \\
\hline $\mathrm{CP}$ & 3378 & 2.8 & $(2.2)$ & 2 & 12.6 & (20.3) & 9 \\
\hline UCLP & 1881 & 4.0 & (1.9) & 4 & 16.1 & $(16.8)$ & 13 \\
\hline BCLP & 881 & 4.7 & (2.1) & 4 & 19.7 & (15.5) & 16 \\
\hline All & 8482 & 3.2 & $(2.1)$ & 3 & 13.2 & (17.9) & 10 \\
\hline \multicolumn{8}{|c|}{ (B) Syndromic patients } \\
\hline $\mathrm{CL}$ & 187 & 6.6 & (6.7) & 4 & 55.3 & (121.8) & 18 \\
\hline $\mathrm{CP}$ & 1827 & 6.4 & $(6.2)$ & 5 & 48.5 & (64.6) & 28 \\
\hline UCLP & 215 & 7.5 & (5.4) & 5 & 58.5 & $(88.2)$ & 26 \\
\hline BCLP & 181 & 8.8 & (6.3) & 7 & 67.5 & (94.3) & 35 \\
\hline All & 2410 & 6.7 & (6.2) & 5 & 51.4 & (75.5) & 27 \\
\hline
\end{tabular}


Table 2 Total number of cleft-related procedure admissions and days in hospital for (A) non-syndromic and (B) syndromic cleft patients in the first 2 years of life, according to cleft type; year of birth 1997-2008

\begin{tabular}{|c|c|c|c|c|}
\hline & & $\begin{array}{l}\text { Total number } \\
\text { admissions }\end{array}$ & & $\begin{array}{l}\text { Total number of days in } \\
\text { hospital }\end{array}$ \\
\hline Cleft type & $\mathrm{N}^{*}$ & Mean (SD) & Median & Mean (SD) \\
\hline
\end{tabular}

(A) Non-syndromic patients

\begin{tabular}{lrrrrrrr} 
CL & 2326 & 1.2 & $(0.4)$ & 1 & 4.0 & $(2.0)$ & 4 \\
CP & 3004 & 1.2 & $(0.5)$ & 1 & 5.0 & $(3.8)$ & 4 \\
UCLP & 1877 & 2.3 & $(0.7)$ & 2 & 9.0 & $(4.4)$ & 8 \\
BCLP & 880 & 2.6 & $(0.9)$ & 2 & 11.2 & $(8.5)$ & 10 \\
All & $8087 \dagger$ & 1.6 & $(0.8)$ & 1 & 6.3 & $(5.0)$ & 5 \\
(B) Syndromic patients & & & & & & \\
CL & 180 & 1.4 & $(0.7)$ & 1 & 11.0 & $(40.0)$ & 4 \\
CP & 1623 & 1.2 & $(0.5)$ & 1 & 6.4 & $(12.7)$ & 5 \\
UCLP & 211 & 2.2 & $(0.8)$ & 2 & 13.7 & $(23.7)$ & 9 \\
BCLP & 180 & 2.6 & $(1.0)$ & 2 & 19.1 & $(43.3)$ & 11 \\
All & 2194 & 1.5 & $(0.8)$ & 1 & 8.5 & $(21.7)$ & 5 \\
\hline
\end{tabular}

Hospital Episode Statistics, England 1997-2011.

*Private, non-UK patients are excluded.

tNot all patients included in the analyses had a cleft-related procedure admission within the first 2 years of life.

$B C L P$, bilateral cleft lip and palate; CL, cleft lip; CP, cleft palate; UCLP, unilateral cleft lip and plate.

with additional anomalies. The number of individual admissions for cleft-related procedures has not changed over time.

Overall, there were 16111 cleft-related admissions, accounting for $37.2 \%$ of all hospital admissions. These admissions resulted in 69675 days, which represents $29.6 \%$ of the total number of days spent in hospital among children with a cleft in the first 2 years of life. Children without additional anomalies spent on average 6.3 days in hospitals for cleft-related care and children with additional anomalies 8.5 days (table 2). Differences in admissions and total time in hospital for cleft-related care were observed between cleft types, again with the more severe cleft types linked to more admissions and longer duration of hospital stay. The difference between the children with and without additional anomalies is relatively small.

\section{Emergency admissions}

In total, there were 11385 emergency admissions, representing $26.3 \%$ of all admissions and $32.3 \%$ of all non-birth admissions. There were 55189 days in hospital resulting from these emergency admissions, representing $23.4 \%$ of the total time spent in hospital in the first 2 years. A total of 4606 (42.3\%) children had at least one emergency admission. These children spent on average 12.0 days in hospital as part of emergency/unplanned admissions (table 3). There was a marked difference between the children with and without additional anomalies; $35.2 \%$ of the children without and $67.3 \%$ of children with additional anomalies had at least one emergency admission. The number of emergency admissions did not vary according to cleft type.

\section{Emergency readmissions}

A total of $558(5.1 \%)$ children had an emergency readmission within 14 days of discharge after a primary cleft repair. Almost twice as many children with additional anomalies had an emergency readmission (7.9\%) as those without (4.3\%). Emergency readmissions also varied according to cleft type. For example,
Table 3 Total number of all-cause 'emergency' admissions and days in hospital for patients in the first 2 years of life, according to syndrome status; year of birth 1997-2008

\begin{tabular}{|c|c|c|c|c|c|c|c|}
\hline \multirow{2}{*}{$\begin{array}{l}\text { Syndrome } \\
\text { status }\end{array}$} & \multirow[b]{2}{*}{$\mathrm{N}^{*}$} & \multicolumn{3}{|c|}{$\begin{array}{l}\text { Total number of } \\
\text { emergency admissions }\end{array}$} & \multicolumn{3}{|c|}{$\begin{array}{l}\text { Total number of days in } \\
\text { hospital }\end{array}$} \\
\hline & & Mean & (SD) & Median & Mean & (SD) & Median \\
\hline Non-syndromic & 2984 & 1.7 & $(0.6)$ & 1 & 5.9 & $(16.5)$ & 3 \\
\hline Syndromic & 1622 & 3.8 & $(4.2)$ & 2 & 23.2 & (41.7) & 9 \\
\hline All & 4606 & 2.5 & (3.0) & 1 & 12.0 & (29.3) & 4 \\
\hline
\end{tabular}

among children without additional anomalies, readmission rates were lowest among children with a CP (2.3\%) and highest in those with a BCLP $(9.8 \%)$. There was a range of causes for the emergency readmissions, with the three most common reported as 'complications of procedures' (22.2\%), 'acute upper respiratory infections of multiple and unspecified sites' (9.9\%) and 'abnormalities of breathing' (7.4\%).

\section{DISCUSSION}

This study found that, on average, children with a cleft and no additional anomalies had three admissions and spent around 2 weeks in hospital before their second birthday. Children with additional anomalies had twice as many admissions, spent between two and three times as long in hospital, and they had more all-cause emergency admissions and emergency readmissions after cleft surgery than children with a cleft alone. Using HES and birth registry data, we calculated that all children born in England, including those with and without clefts, have an average of 1.5 admissions and 3.4 days in hospital in the first 2 years of life.

Admissions and total time in hospital for cleft-related procedures were similar between those with and without additional anomalies. Finally, our study found that admissions and total length of stay were clearly linked to cleft type classification.

Our results resemble those of a US study carried out in Massachusetts in children born 1998-2002, which reported that children with a cleft and additional anomalies stayed at least three times as long in hospital as children without other anomalies, despite a shorter overall hospital stay in the USA. ${ }^{9}$

The present study found only small differences in mean admissions and total time in hospital for cleft-related procedures between children without and those with additional anomalies, which suggests that the extra all-cause admissions and time in hospital observed among those with additional anomalies can be attributed to care not directly related to the cleft.

As expected, we found that children with a CL as well as a $\mathrm{CP}$ spent more time in hospital than children with a CL or a CP. This is similar to results of a Danish study that looked at hospital use in patients with a cleft across their life span. ${ }^{10}$ Increasing time in hospital with increasing severity of cleft type is not surprising given the increasing potential for postoperative complications with increasing surgical complexity. Indeed, our findings showed four times as many BCLP patients as CP patients had an emergency readmission after a primary cleft repair.

Approximately 2\% of children who underwent a primary lip repair were discharged on the same day. This is substantially lower than the $27.9 \%$ reported in a study of 23 US Children's hospitals, which reported a $2.8 \%$ 48-h readmission rate for 
those discharged the same day as their lip repair. ${ }^{11}$ Differences in the way healthcare is funded between the UK and the USA may, in part, explain the higher same-day discharge rates in the USA. Previous research has shown that patients who were discharged on the same day as their primary lip repair in the USA did not have higher readmission rates than those who stayed in hospital, providing patients did not have pre-existing comorbidities. ${ }^{12} 13$ We are aware that some cleft centres in England have recently moved to day-case admissions for cleft lip repair, so the proportion of day-case admissions for this type of repair may be increasing and, thus, the time spent in hospital decreasing.

The drive to reduce length of stay in hospital comes with the growing emphasis on cost reduction and increased efficiency in healthcare services. Our analyses revealed a trend for reduced total length of stay for cleft-related procedures over the 12-year period of births examined, which coincides with the process of centralisation of cleft services in England. ${ }^{5}$

Some English hospitals admit all babies the day before surgery for overnight oxygen saturation and airway monitoring. This inevitably adds an additional day to the duration of hospital stay. While this practice may increase length of stay, clinicians at these sites justify this practice as it may help in detecting children who have a subclinical upper respiratory tract infection. In one hospital, overnight home saturation monitoring for babies before a repair procedure is being trialled.

Our study has an important strength. It is based on a national database that can be expected to include all—or almost allchildren treated for a cleft in England. The data presented are therefore representative of all children treated in England over the 14 years examined.

We were able to examine differences in admissions and total length of stay according to patient and cleft characteristics. Previously, we have explored the accuracy of cleft type diagnostic coding within HES, linking HES data to the CRANE database, a national register that contains information submitted by all hospitals that treat children with a cleft in England, Wales and Northern Ireland. ${ }^{14}$ We found a high level of agreement $(>95 \%)$ in reported cleft type between HES and the CRANE database, suggesting that HES records are a reliable source of data on cleft type. ${ }^{15}$

A first limitation of our study is that the HES database does not provide any information about procedures carried out in approximately $15 \%$ of all planned admissions. It is not clear whether the absence of a procedure code indicates that, during the admission, no cleft-related procedures were carried out or whether a procedure code was omitted in error. If the latter occurred, our numbers reflecting cleft-related admissions are an underestimate. Also, it is likely that not all cleft-related admissions will have involved specific cleft procedures, which may further reduce our burden of cleft-related hospital care estimates.

Second, the choice of procedure codes for the definition of 'cleft-related procedures' (see online supplementary appendix 1) is not straightforward. OPCS codes at the 3-character level were used for pragmatic reasons as well as to avoid being too prescriptive in our definitions. It is acknowledged that the use of 3-character level codes may lead to the inclusion of a small number of non-cleft-related procedures that could be identified and excluded by consideration of the full 4-character code.

Third, it is possible that cleft care in some sites has changed over the past couple of years. For example, we are aware of a number of attempts in some English cleft centres to reduce the duration of hospital stay, including a move towards same-day admissions for all primary cleft lip repair surgery and routine suture removal in outpatient clinics (personal communications).

\section{Implications}

Quantifying the burden of hospital cleft care is important for the planning and commissioning of cleft services. The total time spent in hospital has a major impact on costs. Discharging a child early after a cleft-related procedure will reduce costs and the risk of hospital-acquired infection, but it may lead to the development of complications outside a hospital, including poor pain and fluid management. ${ }^{16}$

Our results are important for the counselling of parents and carers. ${ }^{6}$ In order to reduce anxiety and uncertainty, clear, consistent and accurate information about the expected treatments should be given during initial counselling. Having information on the likely number of hospital admissions and total time spent in hospital in the first years of life may help to lessen the potential anxiety felt by parents and carers of children with a cleft.

Acknowledgements We thank the Health and Social Care Information Centre for providing the hospital episode statistics data used in this study. The authors also thank the following clinical directors of cleft services in England and Wales for their insightful comments on the final draft of this paper: Adrian W Sugar, Consultant/ Senior Lecturer in Cleft and Maxillofacial Surgery and Clinical Director of the South Wales Cleft Centre, Morriston Hospital, Swansea, Wales; Loshan Kangesu, Consultant Plastic Surgeon and Clinical Director of the North Thames Cleft Centre, Great Ormond Street Hospital, London; Alistair Smyth, Consultant Cleft, Oral and Maxillofacial Surgeon and Clinical Director of the Northern and Yorkshire Cleft Lip and Palate Service, Leeds General Infirmary, Leeds, UK; Peter Hodgkinson,

Consultant Plastic Surgeon and Clinical Director of the Northern and Yorkshire Cleft Service, Royal Victoria Infirmary, Newcastle, UK; Liz Albery, Lead Speech and Language Therapist and Clinical Director of the South West Cleft Service, Frenchay Hospital, Bristol, UK; Per Hall, Lead Surgeon and Clinical Director of the East of England Cleft Network, Addenbrooke's Hospital, NHS Foundation Trust, Cambridge, UK; Rona Slator, Consultant Plastic Surgeon and Clinical Lead of the West Midlands Cleft Service, Birmingham Children's Hospital, Birmingham, UK; Simon van Eeden, Consultant Oral and Maxillofacial Surgeon and Clinical Director of the North West and North Wales Cleft Network, Alder Hey Children's NHS Foundation Trust, Liverpool, UK; Felicity Mehendale, Consultant Cleft and Plastic Surgeon and Clinical Lead for the East of Scotland Cleft Lip and Palate Service, Royal Hospital for Sick Children, Edinburgh, UK.

Contributors KF wrote the first draft of the manuscript. LC acquired the data and performed the analyses. SD and JvdM conceived of the study, participated in its design and coordination and edited the manuscript. All authors read and approved the final manuscript.

Funding This work was funded by the National Specialised Commissioning Group for England and the Wales Specialised Health Services Committee and was carried out by the team of the CRANE Database (the national cleft database for England, Wales and Northern Ireland), which is overseen by the UK NHS Cleft Development Group.

\section{Competing interests None.}

Provenance and peer review Not commissioned; externally peer reviewed.

Data sharing statement This study is based on data from the Hospital Episode Statistics database, which can be accessed through the Health and Social Care Information Centre.

Open Access This is an Open Access article distributed in accordance with the Creative Commons Attribution Non Commercial (CC BY-NC 3.0) license, which permits others to distribute, remix, adapt, build upon this work non-commercially, and license their derivative works on different terms, provided the original work is properly cited and the use is non-commercial. See: http://creativecommons.org/ licenses/by-nc/3.0/

\section{REFERENCES}

1 Stanier P, Moore G. Genetics of cleft lip and palate: syndromic genes contribute to the incidence of non-syndromic clefts. Hum Mol Genet 2004;13:R73-81.

2 CRANE Project team on behalf of the Cleft Development Group. CRANE Database Annual Report 2011. London: Clinical Effectiveness Unit, The Royal College of Surgeons of England, 2011.

3 World Health Organization, Human Genetics Programme. World Atlas of Birth defects, 2nd edn. Geneva: International Centre for Birth Defects (ICBD) of the International Clearinghouse for Birth Defects Monitoring Systems in collaboration with European Surveillance of Congenital Anomalies (EUROCAT) in cooperation with Human Genetics Programme World Health Organization, 2003. 
4 Clinical Standards Advisory Group. Report of a CSAG Committee on cleft lip and/or palate. London: The Stationery Office, 1998.

5 Fitzsimons K, Mukarram S, Copley L, et al. Centralisation of services for children with cleft lip or palate in England: a study of hospital episode statistics. BMC Health Serv Res 2012;12:148.

6 Kuttenberger J, Ohmer JN, Polska E. Initial counselling for cleft lip and palate: Parents' evaluation, needs and expectations. Int J Oral Maxillofac Surg 2010:39:214-20.

7 Hospital Episode Statistics. HES user guide. Health and Social Care Information Centre, 2010. http://www.hscic.gov.uk/hes

8 Office of the Deputy Prime Minister. Index of Multiple Deprivation. 2004.

9 Weiss J, Kotelchuck M, Grosse SD, et al. Hospital use and associated costs of children aged zero-to-two years with craniofacial malformations in Massachusetts. Birth Defects Res A Clin Mol Teratol 2009;85:925-34.

10 Wehby G, Pedersen D, Murray J, et al. The effects of oral clefts on hospital use throughout the lifespan. BMC Health Serv Res 2012;12:58.
11 Hopper RA, Lewis C, Umbdenstock R, et al. Discharge practices, readmission, and serious medical complication following primary cleft lip repair in 23 U.S. children's hospitals. Plast Reconstr Surg 2009:123:1553-9.

12 Eaton AC, Marsh JL, Pilgram TK. Does reduced hospital stay affect morbidity and mortality rates following cleft lip and palate repair in infancy? Plast Reconstr Surg 1994;94:911-5.

13 Rosen H, Barrios LM, Reinisch JF, et al. Outpatient cleft lip repair. Plast Reconstr Surg 2003;112:381-7.

14 The Royal College of Surgeons of England. The CRANE database. London. http:// www.crane-database.org.uk/

15 CRANE Project team on behalf of the Cleft Development Group. CRANE Database Annual Report 2012. London: Clinical Effectiveness Unit, The Royal College of Surgeons of England, 2012.

16 Lees VC, Pigott RW. Early postoperative complications in primary cleft lip and palate surgery-how soon may we discharge patients from hospital? Br J Plasti Surg $1992: 45: 232-4$ 